\title{
The Blood Pressure Paradox in Acute Ischemic Stroke
}

Lan Hong ${ }^{1}$, MD, Xin Cheng ${ }^{1}, \mathrm{MD}, \mathrm{PhD}$, Longting $\mathrm{Lin}^{2}$, $\mathrm{PhD}$, Andrew Bivard ${ }^{3}, \mathrm{PhD}$, Yifeng

Ling $^{1}$, MD, Kenneth Butcher ${ }^{4}$, FRACP, Qiang Dong ${ }^{1}, \mathrm{MD}$, PhD, Mark Parsons ${ }^{3}, \mathrm{PhD}$, FRACP, on behalf of the INSPIRE Study Group

1. Department of Neurology, National Clinical Research Centre for Aging and Medicine, Huashan Hospital, State Key Laboratory of Medical Neurobiology, Fudan University, Shanghai, China

2. School of Medicine and Public Health, University of Newcastle, Newcastle, Australia

3. Brain Centre at Royal Melbourne Hospital, University of Melbourne, Melbourne, Australia

4. Division of Neurology, Department of Medicine, University of Alberta, Edmonton, Canada

\section{Running Head}

Blood Pressure, Collateral Flow and Clinical Outcome

\section{Corresponding author:}

Xin Cheng, MD, $\mathrm{PhD}$

Department of Neurology

Huashan Hospital Fudan University

12 Wulumuqi Zhong Road

Shanghai, 200040 P. R. China.

Tel: 86-21-52887145

This is the author manuscript accepted for publication and has undergone full peer review but has not been through the copyediting, typesetting, pagination and proofreading process, which may lead to differences between this version and the Version of Record. Please cite this article as doi: $10.1002 /$ ana.25428

This article is protected by copyright. All rights reserved. 
Fax: 86-21-52887145

E-mail: chengxin@fudan.edu.cn

or

Qiang Dong, $\mathrm{MD}, \mathrm{PhD}$

Department of Neurology

Huashan Hospital Fudan University

12 Wulumuqi Zhong Road

Shanghai,200040 P. R. China.

Tel: 86-21-52887145

Fax: 86-21-52887145

E-mail: qiang_dong163@163.com

Word Count:

Tile: 44 characters

Running head: 46 characters

Abstract: 233 words

Introduction: 294 words

Discussion: 1276 words

Body of the manuscript: 3465 words

\section{Number of references:}

39 references

\section{Figures and Tables}

There are 3 tables and 1 figure in this manuscript (detailed legends at the bottom of the manuscript):

This article is protected by copyright. All rights reserved. 
Figure 1 Flow diagram for patient selection

Table 1. The association between blood pressure and collateral flow parameters

Table 2. The association between blood pressure and outcomes

Table 3 The association between BP, mRS 0-1, infarct volume growth and DT $>3 \mathrm{~s} / \mathrm{DT}>6 \mathrm{~s}$ ratio in subgroup analysis (multivariate-adjusted)

\section{Abstract: \\ Objective:}

To explore the association of post-stroke baseline blood pressure with cerebral collateral flow and functional outcome in acute ischemic patients with large vessel occlusion/stenosis.

\section{Methods}

Patients identified with large vessel occlusion/stenosis with baseline multimodal computed tomography, follow-up imaging, and complete clinical profiles were included. A 90-day modified Rankin Scale of 0-1 was defined as an excellent functional outcome. Cerebral collateral flow was quantified by the volume ratio of tissue within the delay time $>3$ seconds perfusion lesion with severely delayed contrast transit (delay time $>3$ s/delay time $>6 \mathrm{~s}$ ).

\section{Results}

There were 306 patients included in this study. With every increase of 10mmHg in baseline systolic blood pressure, the odds of achieving an excellent functional outcome decreased by $12 \%$ in multivariate analysis (odds ratio $0.88, \mathrm{P}=0.048$ ). Conversely, increased baseline blood pressure was associated with better collateral flow. In subgroup analysis of 
patients with major reperfusion, higher blood pressure was associated with decreased infarct growth and a better clinical outcome, and vice versa in patients without reperfusion.

\section{Interpretation}

Higher baseline blood pressure in acute ischemic stroke patients with large vessel occlusion/stenosis was associated with better collateral flow. However, for patients without reperfusion, higher baseline blood pressure was associated with increased infarct growth, leading to an unfavorable clinical outcome. The relationship between blood pressure and outcomes is highly dependent on reperfusion and active blood pressure lowering treatment may be inappropriate in acute ischemic stroke patients prior to reperfusion treatment.

\section{Introduction}

There is no consensus on the optimal target for baseline blood pressure (BP) in acute ischemic stroke patients ${ }^{1-3}$. Studies of the association between acute BP and clinical outcomes are contradictory. Positive, negative or U-shaped correlations between BP and outcomes have all been described ${ }^{4-13}$.

In patients with acute ischemic stroke, especially those with large vessel occlusion or a proximal stenosis, higher BP may help sustain collateral perfusion, which may minimize final infarction. However, high BP may also increase the risk of complications, such as brain edema and hemorrhagic transformation ${ }^{14}$. Few studies have explored the impact of BP differences in specific stroke subtypes. For patients with proximal large vessel occlusion who 
underwent endovascular treatment, it has been demonstrated that higher post-stroke BP was associated with increased volume of final infarction and worse clinical outcomes ${ }^{15,16}$. On the contrary, high BP has also been reported to be associated with better collateral flow in stroke patients with large vessel occlusion ${ }^{17}$. None of these prior studies assessed outcomes with respect to reperfusion status.

CT Perfusion (CTP) imaging has been widely used to determine infarct core and penumbra in acute ischemic stroke patients. Cerebral collateral flow can be quantified using CTP imaging data by measuring the volume of tissue with delayed contrast transit instead of conventional CTA collateral grading ${ }^{18-28}$. More severe delay in contrast transit indicates poorer collateral flow. Therefore, the aim of this study was to investigate the association between post-stroke baseline BP, collateral flow, infarct growth, and clinical outcomes in acute ischemic stroke patients with large vessel occlusion/stenosis using baseline CTP to measure collateral flow. We hypothesized that higher baseline blood pressure would be associated with improved collateral flow, and the effect of BP on outcomes would be modified by the degree of subsequent reperfusion.

\section{Methods}

Patient Selection and Measures of Outcomes

Consecutive acute ischemic stroke patients presenting within 12 hours of symptom onset from 7 medical centers globally 1) Huashan Hospital, China; 2) John Hunter Hospital, Australia; 3) Box Hill Hospital, Australia; 4) the University of Alberta Hospital, Canada; 5) Royal Adelaide Hospital, Australia; 6) Gosford Hospital, Australia; 7) The Second Affiliated Hospital of Zhejiang University, China between 2011 and 2017 were prospectively recruited for the International Stroke Perfusion Imaging Registry (INSPIRE). Patients from INSPIRE registry were included in this study if they met all the following criteria: 1) large-vessel 
occlusion/stenosis of anterior circulation (including extracranial or intracranial segment of internal carotid artery, M1/M2 segment of middle cerebral artery and anterior cerebral artery; stenosis was defined as $>50 \%$ stenosis of the vessel caliber), 2) underwent complete baseline multimodal computed tomography (CT) imaging, including non-contrast CT (NCCT), CT Perfusion (CTP), CT Angiography (CTA), and 24-hour follow-up magnetic resonance imaging (MRI) or repeat multimodal CT (to assess reperfusion), and 3) complete baseline clinical profiles and follow-up data, and 4) did not receive any BP treatment before multimodal CT imaging. Clinical stroke severity was assessed at baseline imaging time using the National Institutes of Health Stroke Scale (NIHSS). The BP measured in the emergency room (ER) for the first time after arrival was considered as baseline. Eligible patients were treated with intravenous thrombolysis and/or endovascular therapy according to guidelines and the clinical judgment of the treating neurologist. The functional outcome was assessed by modified Rankin Scale (mRS) at 90 days after stroke, which was determined by a trained operator blind to patient information using a validated telephone script. A mRS score of 0-1 was identified as the primary outcome. Secondary outcomes included infarct volume growth and parenchymal hematoma Type 2 (PH2) as defined in the European Cooperative Acute Stroke Study II $^{29}$. Written informed consent was obtained from each participant as per local approvals. The study was approved by local ethics committees.

\section{Acute multimodal CT Protocol}

Participants recruited in INSPIRE were scanned using either 64, 128-, 256-,320 detector scanners for non-contrast CT, CT angiography and perfusion CT (GE Lightspeed, GE Discovery, GE Lightspeed, Siemens Somatom Definition Flash dual source, Philips Brilliance iCT, and Toshiba Aquilion One). CT angiography with acquisition from aorta arc to vertex was performed immediately after perfusion CT. (Details of different CT scanners were 
provided in Supplementary Table 1)

\section{4h-48h Imaging Protocol}

All MR compatible participants had follow-up imaging at 24-48 hours after stroke onset with a stroke MRI protocol on a 1.5T or 3.0T scanner (Siemens Avanto or Verio). The MRI imaging protocol included diffusion weighted Imaging (DWI), fluid-attenuation inversion recovery (FLAIR) imaging, MR time-of-flight angiography (MRA), and perfusion weighted imaging (PWI). MR-incompatible patients had follow-up CT (NCCT +/- CTP) at 24-48 hours after stroke.

\section{Image Analysis and Collateral Assessment}

All perfusion images were post-processed on the commercial software MIStar (Apollo Medical Imaging Technology, Melbourne, Australia) with single value deconvolution with delay and dispersion correction. Previously validated thresholds were applied to measure the volume of acute hypoperfused lesion (delay time [DT] $>3 \mathrm{~s}$ ), severely hypoperfused lesion (DT>6s) and infarct core volume (relative cerebral blood flow $[\mathrm{rCBF}]<30 \%)^{19,30}$. Penumbra volume was calculated by acute hypoperfused lesion volume minus the infarct core volume.

The volume ratio of DT $>3$ s/DT $>6$ s was used to quantify the collateral flow, with a higher DT $>3 s / D T>6 s$ ratio indicating better collateral flow. Additionally, in order to justify the measurement of collateral flow using $\mathrm{DT}>3 \mathrm{~s} / \mathrm{DT}>6 \mathrm{~s}$ ratio, we also evaluated cerebral collateral flow using CTA collateral grade using the published Miteff Scale ${ }^{18}$.

Final infarct volume (FIV) on follow-up MRI or CT was also measured using planimetric techniques included in the MIStar software package. Infarct growth was determined as the difference between FIV and CTP infarct core at baseline. Reperfusion was defined as $>90 \%$ reduction in DT>3s volume on follow-up CTP or MR Perfusion (MRP) compared to baseline 
CTP or MRP, or modified thrombolysis in cerebral infarction score (mTICI) 2c/3 at end of procedure for patients receiving endovascular treatment.

\section{Statistical Analysis}

Statistical Analysis was performed on STATA v13.0 (StataCorp, Ltd, College Station, TX). A two-tailed $\mathrm{P}<0.05$ was considered as significant. Mean and standard deviation (SD) were used to describe continuous variables if normally distributed, or median and interquartile range (IQR) if otherwise. Percentage was used to describe categorical variables. Differences of baseline characteristics were compared using student's t test or Mann-Whitney test for continuous variables, and $\chi 2$ test or Fisher exact test for categorical variables. Spearman's $\rho$ correlation coefficient was used to assess the correlation between $\mathrm{DT}>3 \mathrm{~s} / \mathrm{DT}>6 \mathrm{~s}$ ratio and CTA collateral grade. Variables with $\mathrm{P}<0.05$ in univariate analyses or with clinical significance were included in multivariate models. Univariate and multivariate logistic regression models were used to estimate the unadjusted or adjusted odds ratio (OR) and its 95\% confidence interval (95\% CI) on an excellent clinical outcome (mRS 0-1) per 10mmHg change of BP. The association between BP parameters, collateral flow and infarct growth was evaluated using univariate and multivariate generalized linear models with log transformation because of the non-normality distribution of the data. The association between baseline BP and the volume of infarct core, DT $>3$ s were additionally estimated to further confirm the relationship between BP and collateral flow. The 95\% CI of the corresponding coefficients were also provided. The potential modifying effect of reperfusion was first tested using interaction analysis with BP. Then subgroup analysis was performed according to reperfusion status for the association between BP, infarct volume growth, collateral flow and outcomes.

\section{Results}

This article is protected by copyright. All rights reserved. 
Between November 2011 to July 2017, a total number of 1518 patients were recruited in the INSPIRE registry. In the registry database, 381 patients were confirmed with large vessel occlusion/stenosis. Seventy-five patients were excluded because of posterior circulation infarction. Therefore, 306 patients were included in the study (Figure1). The basic characteristics, including imaging analysis were shown in Supplementary Table 2. The median [IQR] age was 69.5 [60.0, 79.0] years with median [IQR] baseline NIHSS 12.0 [7.0, 16.0], and 59.8\% (183) were male. The median [IQR] baseline systolic blood pressure (SBP) was 146.0 [132.8,163.5] mmHg, median [IQR] baseline diastolic blood pressure (DBP) 84.0 [75.8, 93.3] mmHg, and median [IQR] baseline mean arterial pressure (MAP) 105.0 [97.0,115.0] mmHg. There were 72.5\% (222) patients with internal carotid artery (ICA) or M1 segment of middle cerebral artery occlusion/stenosis, 79 with M2 occlusion/stenosis and 5 with ACA occlusion. Median [IQR] baseline core volume was 36 [12, 72] mL. Excellent functional outcome at 3 months was achieved in 39.5\% (121) patients. Additionally, DT $>3 s / D T>6$ s was correlated with a CTA collateral grading system (Miteff scale), meaning there was a significant association between better collateral grade on CTA and less severe delay within the perfusion lesion (Spearman's $\rho=0.15$, 95\%CI 0.03- 0.26, $\mathrm{P}=0.02$ ). ${ }^{18}$

\section{Association between BP and collateral flow}

Every increase of $10 \mathrm{mmHg}$ in SBP was associated with an increase in the DT $>3 \mathrm{~s} / \mathrm{DT}>6 \mathrm{~s}$ ratio (multivariate-adjusted coefficient $0.05,95 \%$ CI 0.03 to $0.07, \mathrm{P}<0.001$ ). A similar relationship was also seen between DBP and DT>3s/DT>6s ratio (multivariate adjusted coefficient $0.07,95 \%$ CI 0.04 to $0.11, \mathrm{P}<0.001$ ), as well as MAP and $\mathrm{DT}>3 \mathrm{~s} / \mathrm{DT}>6$ s ratio (multivariate-adjusted coefficient $0.08,95 \%$ CI 0.05 to $0.11, \mathrm{P}<0.001$ ). Thus, higher BP was associated with a smaller proportion of the total perfusion lesion having DT $>6$ seconds, indicating better collateral flow. Additionally, higher BP was correlated with smaller baseline 
CTP infarct core and smaller DT $>3$ s volumes (Table 1). Removing patients with stenosis or endovascular treatment (EVT) did not affect the correlation of higher BP with smaller core and DT lesions (Supplementary Table 3 \& 4).

\section{Association between BP and outcomes}

With every increment of $10 \mathrm{mmHg}$ in SBP, the unadjusted odds of an excellent functional outcome decreased by $16 \%$ (OR $0.84,95 \%$ CI 0.76 to 0.93 , $\mathrm{P}=0.001$ ). After multivariate adjustment, the odds of an excellent outcome decreased by $12 \%$ (OR $0.88,95 \%$ CI 0.78 to 0.995, $\mathrm{P}=0.048$ ). The association remained significant after excluding patients with stenosis (OR=0.88, 95\% CI 0.78 to $0.998, \mathrm{P}=0.047$, Supplementary Table 3). After excluding EVT patients, the odds ratio for excellent outcome was still reduced but with the reduced sample size the $\mathrm{p}$ value was not significant ( $\mathrm{p}=0.11$, Supplementary Table 4). Univariate analysis indicated that higher MAP was also associated with decreased odds of an excellent outcome, but the association disappeared after multivariate analysis (Table 2).

Higher SBP was also associated with increased infarct growth (multivariate-adjusted coefficient 0.009 , 95\% CI 0.006 to $0.012, \mathrm{P}<0.001$ ). This association was not affected by whether final infarct volume was measured with CT or MRI (Supplementary Table 5). Similarly, this association was not affected by excluding patients with stenosis or endovascular treatment (Supplementary Table $3 \&$ 4). The same correlation was observed for DBP and MAP. There was no correlation between higher BP and PH2 (Table 2). However, the rate of PH2 was relatively low (Supplementary Table 2).

Interaction analysis and subgroup analysis according to reperfusion status

Of the 272 patients with follow-up perfusion imaging or DSA, 68 (25\%) had major reperfusion (The comparison of baseline and follow-up data between patients with or without 
follow-up perfusion imaging are provided in Supplementary Table 6).

In the subgroup analysis according to reperfusion status, higher baseline BP was associated with better collateral flow regardless of reperfusion. However, higher BP was correlated with increased infarct growth in patients without reperfusion, but decreased infarct volume growth in patients with reperfusion. Higher BP was associated with decreased odds of an excellent functional outcome only in patients without reperfusion. For every 10mmHg increase in SBP, the odds of an excellent functional outcome were reduced by $16 \%$ (multivariate-adjusted OR $0.84,95 \%$ CI 0.72 to $0.99, \mathrm{P}=0.03$ ). However, for patients with reperfusion, every $10 \mathrm{mmHg}$ increase in DBP was associated with $78 \%$ increased odds of an excellent functional outcome (multivariate-adjusted OR 1.78, 95\%CI 1.004 to 3.15, $\mathrm{P}=0.048$ ). No association was found between BP and PH2 in either group. All the interactions between BP and reperfusion with respect to collateral flow, infarct volume growth and mRS 0-1 after multivariate adjustment were statistically significant (Table 3).

\section{Discussion}

We have observed that higher baseline BP after stroke was associated with improved collateral flow, yet worse clinical outcomes. This paradoxical effect is explained by our additional findings of higher BP leading to greater infarct growth in patients without reperfusion. Notably, higher BP was associated with decreased infarct growth in patients with subsequent reperfusion, presumably due to the improved collateral flow demonstrated. Thus, higher BP may be harmful in patients without reperfusion, but protective (via better collateral flow) in those who do have reperfusion.

Patients with $>50 \%$ large vessel stenosis were also included in this study. Because a considerable number of ischemic strokes in Asian population are caused by chronic artery 
stenosis to occlusion, and the mechanism of stroke may be a combination of chronic stenosis, artery to artery embolization and in situ thrombus formation. Whether or not including patients with stenosis did not affect the associations between BP, collaterals, infarct volume growth and clinical outcomes.

To our knowledge, this is the first time that the association between higher blood pressure and better collateral flow has been confirmed and quantified by perfusion imaging. Jiang et al. studied the association between collateralization and BP using a parameter called "relative filling time delay” (rFTD) ${ }^{17,31}$. This a qualitative method adapted from the Miteff collateral grading scale ${ }^{18}$. The prior study demonstrated that higher BP was associated with shorter rFTD, meaning better collateral flow, which is consistent with our finding. Further, only $56.7 \%$ of that study population were identified with ICA/M1 occlusion/stenosis, and rFTD is a crude estimation only for occlusion in ICA or proximal M1 large vessel occlusion. We used the ratio between the volume of hypoperfused 'penumbral range' tissue (DT>3s) and volume of tissue within the total DT3>s lesion with more severe delay (DT>6s) to evaluate the collateral flow ${ }^{19-26}$. Indeed, Delay Time is essentially a quantification of collateral flow, measuring the time it takes for contrast to arrive in ischemic tissue $e^{27,28}$. We demonstrated that with higher BP less of the total perfusion lesion had DT $>6$ s, indicating improved collateral flow.

Apart from higher DT>3s/DT>6s ratios, increasing BP was also associated with reduced volumes of baseline CTP infarct core and DT $>3$ s lesions. It has been postulated that better collateral flow leads to slower infarct core growth ${ }^{32}$, thus our finding of smaller baseline cores, less severe delay measures of collateral flow, decreased infarct volume growth and better clinical outcomes in reperfusers confirms this theory. Our data are all consistent with the fact 
that higher baseline BP leads to better collateral flow.

Previous studies regarding the relationship between BP and stroke outcomes have shown conflicting results. The Fukuoka Registry demonstrated that high BP was associated with poor functional outcome by averaging BP over the first $48 \mathrm{~h}$ post-stroke ${ }^{4}$. Studies in large vessel occlusion patients with earlier generation endovascular treatment (with less effective reperfusion) also showed that higher admission BP was associated with larger FIV and still decreased odds of a good functional outcome ${ }^{15,16}$. However, a further study demonstrated that lower BP at admission was associated with increased mortality after acute ischemic stroke 9 . As for blood pressure lowering treatment, there is no substantial evidence showing its benefit for acute ischemic stroke patients ${ }^{2,3,33}$ and the ongoing Enhanced Control of Hypertension and Thrombolysis in Stroke Study (ENCHANTED) is exploring the effect of intensive blood pressure lowering treatment ${ }^{34}$. The reason for these contradictory results may partly due to unmeasured modifying effect of subsequent reperfusion on the outcomes in these studies. Our study is the only one to explore the association between BP and outcomes according to reperfusion status and has revealed that it was only in patients without reperfusion that higher BP was associated with less favorable clinical outcomes, probably accounted for by increased infarct growth. Conversely, for patients achieving reperfusion, higher baseline BP was associated with decreased infarct volume growth. Though the reperfusion rate was only $25 \%$ (due to the low rate of endovascular therapy) in our study, we managed to demonstrate that higher baseline BP was associated with a better clinical outcome in patients with reperfusion. Meanwhile, any differential effect of SBP, DBP and MBP on outcomes needs further investigation.

Therefore, our study suggests that the 'paradox' of high blood pressure leading to smaller 
infarct core in acute ischemic stroke lies in the modifying effect of reperfusion. Initially, higher baseline blood pressure may improve collateral flow to sustain penumbra and prevent the core from expanding. Patients with good collaterals are likely to be 'slow infarct core growers $^{32}$. In such patients with good collateral flow, if there is subsequent reperfusion there may be smaller infarct volume, and hence better clinical outcomes. However, if the occlusion persists and reperfusion does not occur, eventually the infarct core will grow (despite good collaterals), leading to a worse clinical outcome.

Alternately, our data may indicate that in clinical practice, unless major reperfusion occurs, higher BP may be harmful. Previous trials performed before multimodal imaging testing hypervolemic treatment failed to show any significant benefit ${ }^{35,36}$. However, in the context of our study, BP raising treatments (such as hypervolemia or pressor agents) may be worth revisiting in LVO patients, with the caveat that they should be ceased following reperfusion.

There are several limitations of our study. First, this study only explored the influence of baseline BP (BP measured for the first time in ER) on outcome. The measurement of BP in the ER might not follow the most recommended standard, which probably had influence on the analysis. The course of BP over time and the treatment of BP may also have an impact on collateral flow and outcomes. Further studies may explore the effects of BP for the first 24 hours after admission. BP treatment after reperfusion should also be adjusted as confounders. Secondly, we were unable to determine the mechanism of the relationship between higher BP and worse outcomes in patients without reperfusion. Notably, PH2 was not associated with higher BP in this study, but this may reflect the low number of PH2 cases. The relationship between higher BP and brain edema is also worth investigating. Since brain edema usually peaks at 3-5 days after stroke ${ }^{37}$, the 24-48 hour follow-up imaging in our study might not be 
suitable for edema assessment. Nonetheless, the association between BP and edema may confound the effect of association between BP and infarct volume growth. Thirdly, different CT scanners were used in the study for CT perfusion imaging, which may have slight influence on the analysis. However, it also makes the results more generalizable and previous INSPIRE studies have shown that the thresholds for core and penumbra are robust across different CT scanners. Fourth, reperfusion status was only assessed once at a delayed timepoint after reperfusion therapy, thus it is not possible to be sure when these patients actually reperfused within the first 24 hours. Finally, this is the first time that the $\mathrm{DT}>3 \mathrm{~s} / \mathrm{DT}>6 \mathrm{~s}$ ratio has been proposed as a marker of collateral flow. We did find that the DT $>3 s / D T>6 s$ ratio correlated with the Miteff collateral scale on CTA. Although the strength of the association was not very strong, this likely reflects that grading of collaterals on CTA has its own limitations, and we believe that automated CTP measures of delay allow quantification of collateral flow, as opposed to subjective qualitative assessments on CTA. Notably, a similar CTP measure, hypoperfusion intensity ratio (HIR), defined as the volume ratio of Tmax (Time to maximum tissue residue function) $>10 \mathrm{~s} / \mathrm{Tmax}>6 \mathrm{~s}$, has also recently been proposed as a measure of collateral flow ${ }^{38}$. Our reasoning behind using Delay Time rather than Tmax relates to our previous studies that have shown DT provides an assessment of hypoperfused tissue at least as accurate as Tmax. ${ }^{27,28,39}$

\section{Conclusion}

Higher baseline blood pressure was associated with better collateral flow for acute ischemic stroke patients with large vessel occlusion/stenosis. Improved collateral flow in patients with higher BP did not translate into better clinical outcomes. This seemingly paradoxical result may be explained by the additional association of higher BP with decreased infarct growth and favorable clinical outcomes in patients with reperfusion, but greater infarct 
growth and worse clinical outcomes in patients without subsequent reperfusion

\section{Acknowledgements}

This study is funded by National Key R\&D Program of China (2017YFC1308201), grant from Science and Technical committee of Shanghai Municipality (No. 124119a8100) and National Health and Medical Research Council of Australia Project Grant (ID 1013719).

\section{Author Contributions}

XC, MP and QD contributed to conception and design of the study.

LH, LL, AB, YL and KB contributed to the analysis of the data.

LH contributed to drafting the text and preparing the tables and figures.

The authors below were members of the INSPIRE Study Group participating in data collection, critical review and revision of this manuscript:

Neil Spratt, PhD, FRACP, Department of Neurology, John Hunter Hospital, Newcastle, Australia

Christopher Levi, FRACP, Department of Neurology, John Hunter Hospital, Newcastle, Australia

Philip M.C. Choi, FRACP, Department of Neurosciences, Eastern Health, Box Hill, Melbourne, Australia

Timothy Kleining, PhD, FRACP, Department of Neurology, Royal Adelaide Hospital, Adelaide, Australia

Billy O’Brien, FRACP, Department of Neurology, Gosford Hospital, Gosford, Australia Min Lou, PhD, FRACP, Department of Neurology, the Second Affiliated Hospital of Zhejiang University, Hangzhou, China 


\section{Potential Conflicts of Interest}

None

\section{Reference}

1. Powers WJ, Rabinstein AA, Ackerson T, et al. 2018 Guidelines for the Early Management of Patients With Acute Ischemic Stroke: A Guideline for Healthcare Professionals From the American Heart Association/American Stroke Association. Stroke 2018;49:e46-e110.

2. He J, Zhang YH, Xu T, et al. Effects of Immediate Blood Pressure Reduction on Death and Major Disability in Patients With Acute Ischemic Stroke The CATIS Randomized Clinical Trial. JAMA 2014;311:479-89.

3. Sandset EC, Bath PM, Boysen G, et al. The angiotensin-receptor blocker candesartan for treatment of acute stroke (SCAST): a randomised, placebo-controlled, double-blind trial. Lancet 2011;377:741-750.

4. Ishitsuka K, Kamouchi M, Hata J, et al. High Blood Pressure After Acute Ischemic Stroke Is Associated With Poor Clinical Outcomes Fukuoka Stroke Registry. Hypertension 2014;63:54-60.

5. Tziomalos K, Giampatzis V, Bouziana SD, et al. Elevated diastolic but not systolic blood pressure increases mortality risk in hypertensive but not normotensive patients with acute ischemic stroke. Am J Hypertens 2015;28:765-771.

6. Berge E, Cohen G, Lindley RI, et al. Effects of Blood Pressure and Blood Pressure-Lowering Treatment During the First 24 Hours Among Patients in the Third International Stroke Trial of Thrombolytic Treatment for Acute Ischemic Stroke. Stroke 2015;46:3362-3369.

7. Willmot M, Leonardi-Bee J, Bath PM. High blood pressure in acute stroke and subsequent outcome: a systematic review. Hypertension 2004;43:18-24.

8. Mattle HP, Kappeler L, Arnold M, et al. Blood pressure and vessel recanalization in the first hours after ischemic stroke. Stroke 2005;36:264-268.

9. Wohlfahrt P, Krajcoviechova A, Jozifova M, et al. Low blood pressure during the acute period of ischemic stroke is associated with decreased survival. J Hypertens 2015;33:339-345.

10. Leonardi-Bee J, Bath PM, Phillips SJ,et al. Blood pressure and clinical outcomes in the International Stroke Trial. Stroke 2002;33:1315-1320.

11. Castillo J, Leira R, Garcia MM, et al. Blood pressure decrease during the acute phase of ischemic stroke is associated with brain injury and poor stroke outcome. Stroke 2004;35:520-526.

12. Ahmed N, Wahlgren N, Brainin M, et al. Relationship of blood pressure, antihypertensive therapy, and outcome in ischemic stroke treated with intravenous thrombolysis: retrospective analysis from Safe Implementation of Thrombolysis in Stroke-International Stroke Thrombolysis Register (SITS-ISTR). Stroke 2009;40:2442-2449.

13. Manning LS, Rothwell PM, Potter JF, Robinson TG. Prognostic Significance of Short-Term Blood 
Pressure Variability in Acute Stroke: Systematic Review. Stroke 2015;46:2482-2490.

14. Regenhardt RW, Das AS, Stapleton CJ, et al. Blood Pressure and Penumbral Sustenance in Stroke from Large Vessel Occlusion. Front Neurol 2017;8:317.

15. Goyal N, Tsivgoulis G, Iftikhar S, et al. Admission systolic blood pressure and outcomes in large vessel occlusion strokes treated with endovascular treatment. J Neurointerv Surg 2017;9:451-454.

16. Goyal N, Tsivgoulis G, Pandhi A, et al. Blood pressure levels post mechanical thrombectomy and outcomes in large vessel occlusion strokes. Neurology 2017;89:540-547.

17. Jiang BS, Churilov L, Kanesan L, et al. Blood Pressure May Be Associated with Arterial Collateralization in Anterior Circulation Ischemic Stroke before Acute Reperfusion Therapy. J Stroke 2017;19:222-228.

18. Miteff F, Levi CR, Bateman GA, Spratt N, et al. The independent predictive utility of computed tomography angiographic collateral status in acute ischaemic stroke. Brain 2009;132:2231-2238.

19. Bivard A, Levi C, Krishnamurthy V, et al. Defining acute ischemic stroke tissue pathophysiology with whole brain CT perfusion. J Neuroradiol 2014;41:307-315.

20. Chen C, Bivard A, Lin L, et al. Thresholds for infarction vary between gray matter and white matter in acute ischemic stroke: A CT perfusion study. J Cereb Blood Flow Metab 2017; DOI: 10.1177/0271678X177444453

21. Chen C, Parsons MW, Clapham M, et al. Influence of Penumbral Reperfusion on Clinical Outcome Depends on Baseline Ischemic Core Volume. Stroke 2017;48:2739-2745.

22. Bivard A, Kleinig T, Miteff F, et al. Ischemic core thresholds change with time to reperfusion: A case control study. Ann Neurol 2017;82:995-1003.

23. Parsons MW, Pepper EM, Bateman GA, et al. Identification of the penumbra and infarct core on hyperacute noncontrast and perfusion CT. Neurology 2007;68:730-736.

24. Anderson CS, Heeley E, Huang Y, et al. Rapid blood-pressure lowering in patients with acute intracerebral hemorrhage. N Engl J Med 2013;368:2355-2365.

25. Beard DJ, McLeod DD, Logan CL, et al. Intracranial pressure elevation reduces flow through collateral vessels and the penetrating arterioles they supply. A possible explanation for 'collateral failure' and infarct expansion after ischemic stroke. J Cereb Blood Flow Metab 2015;35:861-872.

26. Parsons M, Spratt N, Bivard A, et al. A randomized trial of tenecteplase versus alteplase for acute ischemic stroke. N Engl J Med 2012;366:1099-1107.

27. Bivard A, Levi C, Spratt N, Parsons M. Perfusion CT in acute stroke: a comprehensive analysis of infarct and penumbra. Radiology 2013;267:543-550.

28. Lin L, Bivard A, Kleinig T, et al. Correction for Delay and Dispersion Results in More Accurate Cerebral Blood Flow Ischemic Core Measurement in Acute Stroke. Stroke 2018;49:924-930.

29. Hacke W, Kaste M, Fieschi C, et al. Randomised double-blind placebo-controlled trial of thrombolytic therapy with intravenous alteplase in acute ischaemic stroke (ECASS II). Second European-Australasian Acute Stroke Study Investigators. Lancet 1998;352:1245-1251. 
30. Bivard A, Levi C, Lin L, et al. Validating a Predictive Model of Acute Advanced Imaging Biomarkers in Ischemic Stroke. Stroke 2017;48:645-650.

31. Cao W, Campbell BC, Dong Q,et al. Relative filling time delay based on CT perfusion source imaging: a simple method to predict outcome in acute ischemic stroke. AJNR Am J Neuroradiol 2014;35:1683-1687.

32. Albers GW. Late Window Paradox. Stroke 2018;49:768-771.

33. Sandset EC, Jusufovic M, Sandset PM,et al. Effects of blood pressure-lowering treatment in different subtypes of acute ischemic stroke. Stroke 2015;46:877-879.

34. Anderson CS, Robinson T, Lindley RI, et al. Low-Dose versus Standard-Dose Intravenous Alteplase in Acute Ischemic Stroke. N Engl J Med 2016;374:2313-2323.

35. Aichner FT, Fazekas F, Brainin M,et al. Hypervolemic hemodilution in acute ischemic stroke: the Multicenter Austrian Hemodilution Stroke Trial (MAHST). Stroke 1998;29:743-749.

36. Rudolf J, Group HESiASS. Hydroxyethyl starch for hypervolemic hemodilution in patients with acute ischemic stroke: a randomized, placebo-controlled phase II safety study. Cerebrovasc Dis 2002;14:33-41.

37. Shaw CM, Alvord EC, Jr., Berry RG. Swelling of the brain following ischemic infarction with arterial occlusion. Arch Neurol 1959;1:161-177.

38. Olivot JM, Mlynash M, Inoue M, et al. Hypoperfusion intensity ratio predicts infarct progression and functional outcome in the DEFUSE 2 Cohort. Stroke 2014;45:1018-1023.

39. Lin L, Bivard A, Krishnamurthy V, et al. Whole-Brain CT Perfusion to Quantify Acute Ischemic Penumbra and Core. Radiology 2016;279:876-887. 
Table 1. The association between BP and collateral flow parameters

This article is protected by copyright. All rights reserved. 


\begin{tabular}{|c|c|c|c|c|c|c|}
\hline & \multicolumn{3}{|c|}{ Unadjusted } & \multicolumn{3}{|c|}{ Multivariate adjusted } \\
\hline & Coefficient & $95 \% \mathrm{CI}$ & $\mathrm{P}$ & Coefficient & $95 \% \mathrm{CI}$ & $\mathrm{P}$ \\
\hline \multicolumn{7}{|l|}{$\mathrm{DT}>3 \mathrm{~s} / \mathrm{DT}>\mathbf{6 s} \mathrm{s}^{\mathrm{a}}$} \\
\hline SBP per 10mmHg & 0.04 & $0.02-0.06$ & $<0.001$ & 0.05 & $0.03-0.07$ & $<0.001$ \\
\hline DBP per 10mmHg & 0.07 & $0.04-0.11$ & $<0.001$ & 0.07 & $0.04-0.11$ & $<0.001$ \\
\hline MAP per $10 \mathrm{mmHg}$ & 0.07 & $0.04-0.10$ & $<0.001$ & 0.08 & $0.05-0.11$ & $<0.001$ \\
\hline \multicolumn{7}{|l|}{ Core volume $^{b}$} \\
\hline SBP per 10mmHg & -0.007 & $-0.01--0.0006$ & 0.03 & -0.02 & $-0.04--0.02$ & $<0.001$ \\
\hline DBP per 10mmHg & -0.02 & $-0.03--0.01$ & $<0.001$ & -0.06 & $-0.07--0.05$ & $<0.001$ \\
\hline MAP per $10 \mathrm{mmHg}$ & -0.07 & $-0.04--0.10$ & $<0.001$ & -0.06 & $-0.07--0.04$ & $<0.001$ \\
\hline \multicolumn{7}{|l|}{ DT $>3$ s lesion ${ }^{c}$} \\
\hline SBP per 10mmHg & -0.004 & $-0.002-0.01$ & 0.07 & -0.005 & $-0.01--0.0006$ & 0.03 \\
\hline DBP per 10mmHg & -0.01 & $-0.002--0.02$ & 0.01 & -0.02 & $-0.03--0.01$ & $<0.001$ \\
\hline MAP per $10 \mathrm{mmHg}$ & -0.01 & $-0.003--0.02$ & 0.005 & -0.02 & $-0.02--0.01$ & $<0.001$ \\
\hline
\end{tabular}

Increasing BP was significantly associated with increase in $\mathrm{DT}>3 \mathrm{~s} / \mathrm{DT}>6 \mathrm{~s}$ ratio, and decrease in core, DT $>3 \mathrm{~s}$, DT $>6 \mathrm{~s}$ volume.

a Multivariate-adjusted for age, sex, baseline NIHSS, baseline glucose, history of hypertension, prior stroke, taking antiplatelet before admission, cause of stroke

b Multivariate-adjusted for age, sex, baseline NIHSS, baseline glucose, history of hypertension, prior stroke, taking antiplatelet before admission, occlusion/stenosis of ICA/M1

c Multivariate-adjusted for age, sex, baseline NIHSS, baseline glucose, history of hypertension, prior stroke, taking antiplatelet before admission, history of dyslipidemia, occlusion/stenosis of ICA/M1

Abbreviations: BP blood pressure; SBP systolic blood pressure; DBP diastolic blood pressure; MAP mean arterial pressure; DT delay time; NIHSS National Institutes of Health Stroke Scale; ICA internal carotid artery; M1 M1 segment of middle cerebral artery 
Table 2. The association between BP and outcomes

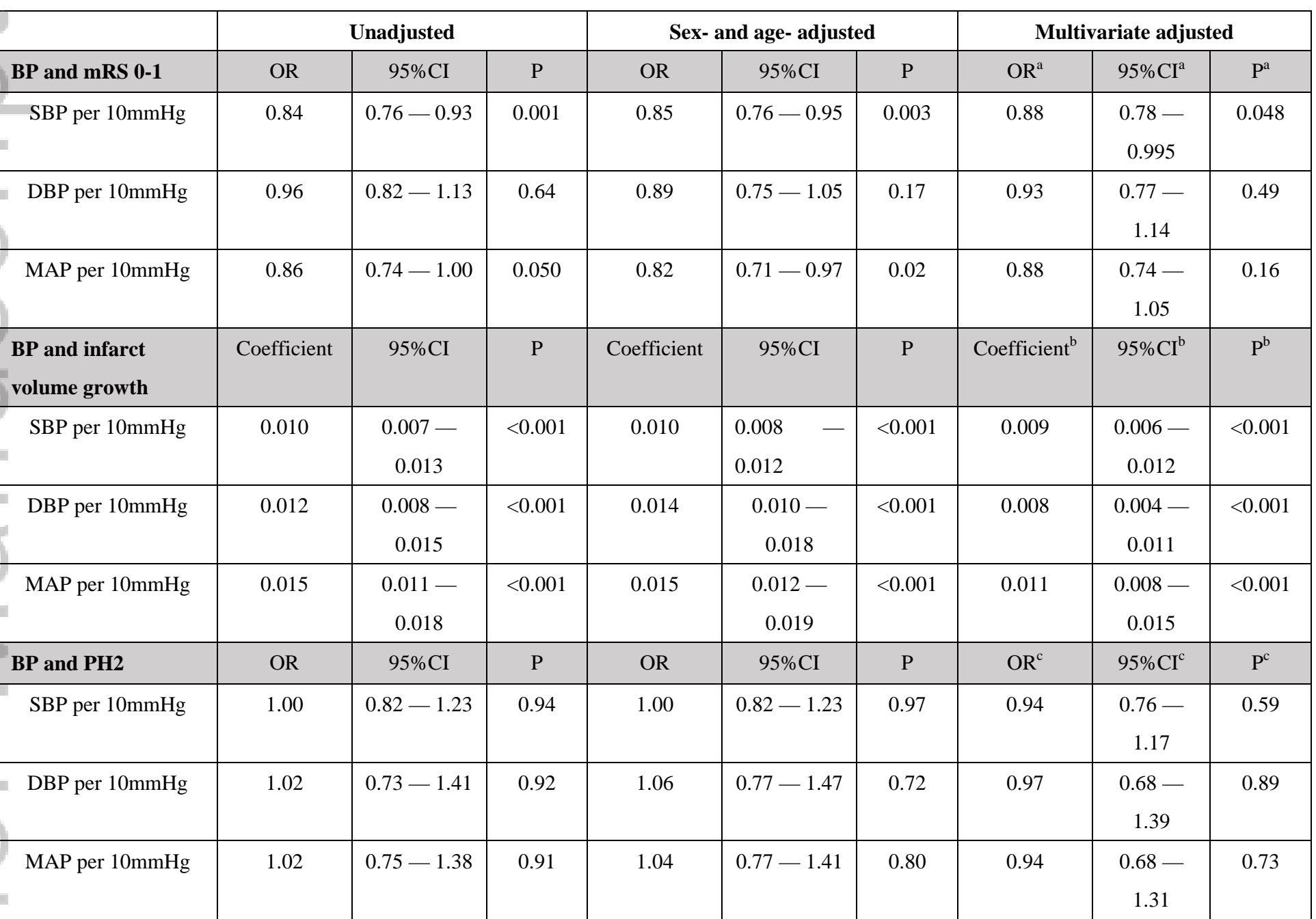

Higher BP was associated with decreased odds of an excellent functional outcome (mRS 0-1) and increased infarct volume growth.

a Adjusted for age, sex, baseline NIHSS, history of hypertension, prior stroke, taking antiplatelet before admission, reperfusion therapy, ischemic core volume, $\mathrm{PH} 2$

b Adjusted for age, sex, baseline NIHSS, baseline glucose, endovascular treatment, occlusion/stenosis of ICA/M1, baseline ischemic core volume, baseline ischemic penumbra volume, MRI vs NCCT

${ }^{\mathrm{c}}$ Adjusted for age, sex, baseline NIHSS, taking antiplatelet before admission, reperfusion therapy

Abbreviations: BP blood pressure; mRS modified Rankin Scale; SBP systolic blood pressure; DBP diastolic blood pressure; MAP mean arterial 
pressure; OR odds ratio; NIHSS National Institutes of Health Stroke Scale; ICA internal carotid artery; M1 M1 segment of middle cerebral artery; PH2 parenchymal hematoma type 2, NCCT Non-Contrast Computed Tomography, MRI Magnetic Resonance Imaging

Table 3. The association between BP, mRS 0-1, infarct volume growth and DT>3s/DT $>6$ s ratio in subgroup analysis (multivariate-adjusted) 


\begin{tabular}{|c|c|c|c|c|c|c|c|}
\hline & \multicolumn{3}{|c|}{ Patients without reperfusion $(\mathrm{n}=204)$} & \multicolumn{3}{|c|}{ Patients with reperfusion(n=68) } & $\mathrm{P}$ for interaction \\
\hline $\begin{array}{l}\mathrm{BP} \text { and } \\
\mathrm{DT}>3 \mathrm{~s} / \mathrm{DT}>6 \mathrm{~s}^{\mathrm{a}}\end{array}$ & Coefficient & $95 \% \mathrm{CI}$ & $\mathrm{P}$ & Coefficient & $95 \% \mathrm{CI}$ & $\mathrm{P}$ & \\
\hline SBP per $10 \mathrm{mmHg}$ & 0.03 & $0.004-0.057$ & 0.02 & 0.08 & $0.04-0.12$ & $<0.001$ & 0.02 \\
\hline DBP per $10 \mathrm{mmHg}$ & 0.03 & $-0.03-0.05$ & 0.63 & 0.13 & $0.05-0.20$ & 0.001 & $<0.001$ \\
\hline MAP per $10 \mathrm{mmHg}$ & 0.03 & $-0.01-0.07$ & 0.13 & 0.14 & $0.07-0.21$ & $<0.001$ & $<0.001$ \\
\hline $\begin{array}{l}\text { BP and infarct } \\
\text { volume growth }^{\mathrm{b}}\end{array}$ & Coefficient & $95 \% \mathrm{CI}$ & $\mathrm{P}$ & Coefficient & $95 \% \mathrm{CI}$ & $\mathrm{P}$ & \\
\hline SBP per 10mmHg & 0.01 & $0.007-0.013$ & $<0.001$ & 0.003 & $-0.003-0.008$ & 0.85 & 0.001 \\
\hline DBP per $10 \mathrm{mmHg}$ & 0.01 & $0.006-0.016$ & $<0.001$ & -0.014 & $-0.025--0.004$ & 0.01 & $<0.001$ \\
\hline MAP per $10 \mathrm{mmHg}$ & 0.013 & $0.009-0.018$ & $<0.001$ & -0.005 & $-0.01--0.004$ & 0.26 & $<0.001$ \\
\hline BP and $m R S ~ 0-1^{c}$ & OR & $95 \% \mathrm{CI}$ & $\mathrm{P}$ & OR & $95 \% \mathrm{CI}$ & $\mathrm{P}$ & \\
\hline SBP per $10 \mathrm{mmHg}$ & 0.84 & $0.72-0.99$ & 0.03 & 0.95 & $0.71-1.29$ & 0.76 & 0.30 \\
\hline DBP per $10 \mathrm{mmHg}$ & 0.83 & $0.65-1.06$ & 0.14 & 1.78 & $1.004-3.15$ & 0.048 & 0.04 \\
\hline MAP per $10 \mathrm{mmHg}$ & 0.80 & $0.63-1.00$ & 0.05 & 1.38 & $0.82-2.36$ & 0.23 & 0.052 \\
\hline
\end{tabular}

For patients without reperfusion, higher BP was associated with better collateral flow, but increased infarct volume growth, and decreased odds of mRS 0-1.

For patients with reperfusion, higher BP was associated with better collateral flow, decreased infarct volume growth and increased odds of mRS 0-1.

Multivariate-adjusted for age, sex, baseline NIHSS, baseline glucose, history of hypertension, prior stroke, taking antiplatelet before admission, cause of stroke

Adjusted for age, sex, baseline NIHSS, baseline glucose, history of hypertension, endovascular treatment, occlusion/stenosis of ICA/M1, baseline ischemic core volume, baseline ischemic penumbra volume, MRI vs NCCT

${ }^{c}$ Adjusted for age, sex, baseline NIHSS, history of hypertension, prior stroke, taking antiplatelet before admission, reperfusion therapy, ischemic core volume, $\mathrm{PH} 2$

Abbreviations: BP blood pressure; SBP systolic blood pressure; DBP diastolic blood pressure; MAP mean arterial pressure; DT delay time; mRS modified Rankin Scale; NIHSS National Institutes of Health Stroke Scale; ICA internal carotid artery; M1 M1 segment of middle cerebral artery; PH2 parenchymal hematoma type 2, NCCT Non-Contrast Computed Tomography, MRI Magnetic Resonance Imaging 
Table 1. The association between BP and collateral flow parameters

\begin{tabular}{|c|c|c|c|c|c|c|}
\hline & \multicolumn{3}{|c|}{ Unadjusted } & \multicolumn{3}{|c|}{ Multivariate adjusted } \\
\hline & Coefficient & $95 \% \mathrm{CI}$ & $\mathrm{P}$ & Coefficient & $95 \% \mathrm{CI}$ & $\mathrm{P}$ \\
\hline \multicolumn{7}{|l|}{$\mathrm{DT}>3 \mathrm{~s} / \mathrm{DT}>6 \mathrm{~s}^{\mathrm{a}}$} \\
\hline SBP per 10mmHg & 0.04 & $0.02-0.06$ & $<0.001$ & 0.05 & $0.03-0.07$ & $<0.001$ \\
\hline DBP per 10mmHg & 0.07 & $0.04-0.11$ & $<0.001$ & 0.07 & $0.04-0.11$ & $<0.001$ \\
\hline MAP per $10 \mathrm{mmHg}$ & 0.07 & $0.04-0.10$ & $<0.001$ & 0.08 & $0.05-0.11$ & $<0.001$ \\
\hline \multicolumn{7}{|l|}{ Core volume $^{b}$} \\
\hline SBP per 10mmHg & -0.007 & $-0.01--0.0006$ & 0.03 & -0.02 & $-0.04--0.02$ & $<0.001$ \\
\hline DBP per 10mmHg & -0.02 & $-0.03--0.01$ & $<0.001$ & -0.06 & $-0.07--0.05$ & $<0.001$ \\
\hline MAP per $10 \mathrm{mmHg}$ & -0.07 & $-0.04--0.10$ & $<0.001$ & -0.06 & $-0.07--0.04$ & $<0.001$ \\
\hline \multicolumn{7}{|l|}{ DT $>3$ s lesion ${ }^{c}$} \\
\hline SBP per 10mmHg & -0.004 & $-0.002-0.01$ & 0.07 & -0.005 & $-0.01--0.0006$ & 0.03 \\
\hline DBP per 10mmHg & -0.01 & $-0.002--0.02$ & 0.01 & -0.02 & $-0.03--0.01$ & $<0.001$ \\
\hline MAP per $10 \mathrm{mmHg}$ & -0.01 & $-0.003--0.02$ & 0.005 & -0.02 & $-0.02--0.01$ & $<0.001$ \\
\hline
\end{tabular}

Increasing BP was significantly associated with increase in $\mathrm{DT}>3 \mathrm{~s} / \mathrm{DT}>6 \mathrm{~s}$ ratio, and decrease in core, DT $>3 \mathrm{~s}$, DT $>6 \mathrm{~s}$ volume.

a Multivariate-adjusted for age, sex, baseline NIHSS, baseline glucose, history of hypertension, prior stroke, taking antiplatelet before admission, cause of stroke

b Multivariate-adjusted for age, sex, baseline NIHSS, baseline glucose, history of hypertension, prior stroke, taking antiplatelet before admission, occlusion/stenosis of ICA/M1

c Multivariate-adjusted for age, sex, baseline NIHSS, baseline glucose, history of hypertension, prior stroke, taking antiplatelet before admission, history of dyslipidemia, occlusion/stenosis of ICA/M1

Abbreviations: BP blood pressure; SBP systolic blood pressure; DBP diastolic blood pressure; MAP mean arterial pressure; DT delay time; NIHSS National Institutes of Health Stroke Scale; ICA internal carotid artery; M1 M1 segment of middle cerebral artery 
Table 2. The association between BP and outcomes

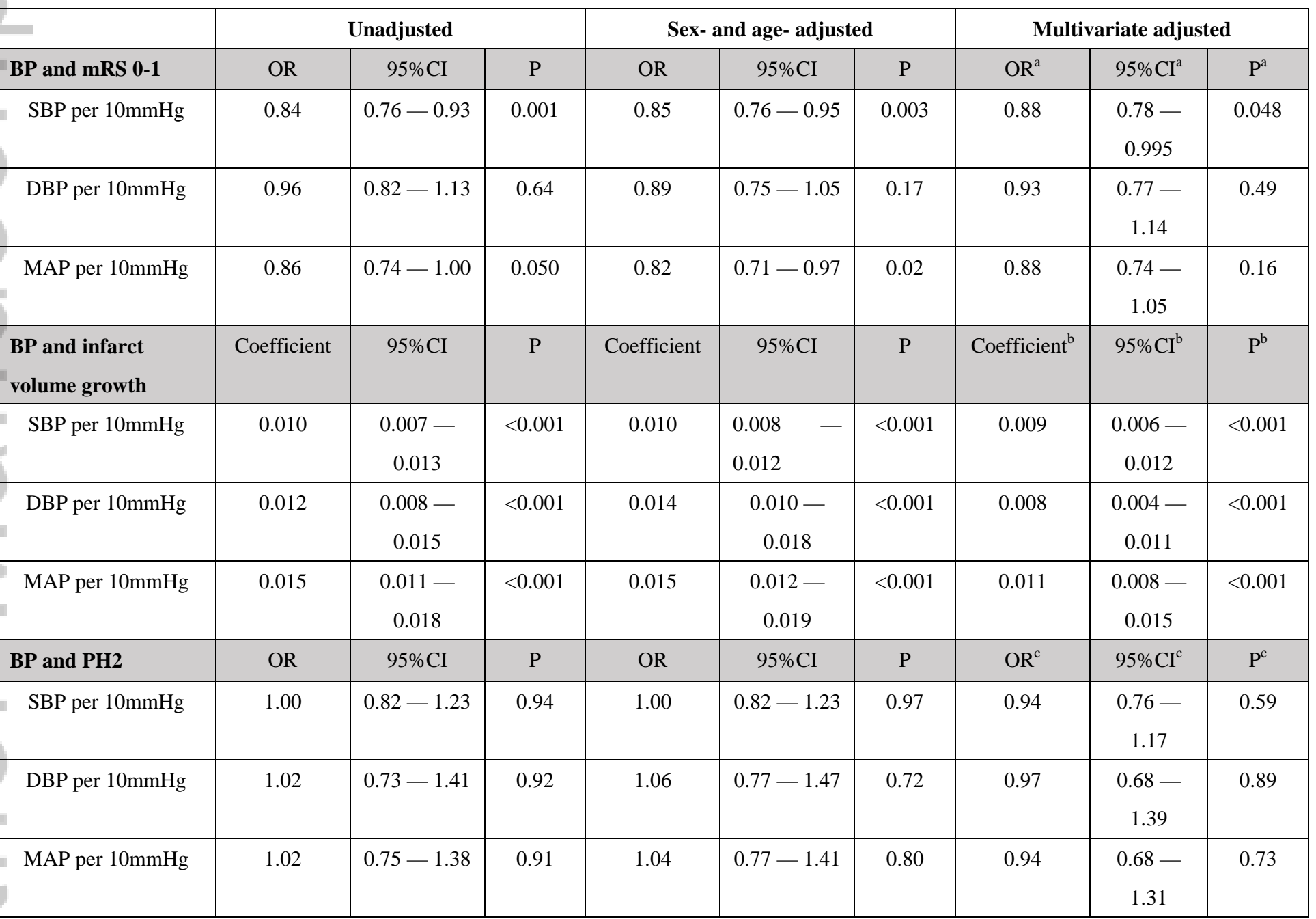

Higher BP was associated with decreased odds of an excellent functional outcome (mRS 0-1) and increased infarct volume growth.

a Adjusted for age, sex, baseline NIHSS, history of hypertension, prior stroke, taking antiplatelet before admission, reperfusion therapy, ischemic core volume, $\mathrm{PH} 2$

${ }^{\mathrm{b}}$ Adjusted for age, sex, baseline NIHSS, baseline glucose, endovascular treatment, occlusion/stenosis of ICA/M1, baseline ischemic core volume, baseline ischemic penumbra volume, MRI vs NCCT 
${ }^{\mathrm{c}}$ Adjusted for age, sex, baseline NIHSS, taking antiplatelet before admission, reperfusion therapy

Abbreviations: BP blood pressure; mRS modified Rankin Scale; SBP systolic blood pressure; DBP diastolic blood pressure; MAP mean arterial pressure; OR odds ratio; NIHSS National Institutes of Health Stroke Scale; ICA internal carotid artery; M1 M1 segment of middle cerebral artery;

PH2 parenchymal hematoma type 2, NCCT Non-Contrast Computed Tomography, MRI Magnetic Resonance Imaging

Table 3. The association between BP, mRS 0-1, infarct volume growth and DT>3s/DT>6s ratio in subgroup analysis (multivariate-adjusted) 


\begin{tabular}{|c|c|c|c|c|c|c|c|}
\hline & \multicolumn{3}{|c|}{ Patients without reperfusion $(\mathrm{n}=204)$} & \multicolumn{3}{|c|}{ Patients with reperfusion(n=68) } & $\mathrm{P}$ for interaction \\
\hline $\begin{array}{l}\mathrm{BP} \text { and } \\
\mathrm{DT}>3 \mathrm{~s} / \mathrm{DT}>6 \mathrm{~s}^{\mathrm{a}}\end{array}$ & Coefficient & $95 \% \mathrm{CI}$ & $\mathrm{P}$ & Coefficient & $95 \% \mathrm{CI}$ & $\mathrm{P}$ & \\
\hline SBP per $10 \mathrm{mmHg}$ & 0.03 & $0.004-0.057$ & 0.02 & 0.08 & $0.04-0.12$ & $<0.001$ & 0.02 \\
\hline DBP per $10 \mathrm{mmHg}$ & 0.03 & $-0.03-0.05$ & 0.63 & 0.13 & $0.05-0.20$ & 0.001 & $<0.001$ \\
\hline MAP per $10 \mathrm{mmHg}$ & 0.03 & $-0.01-0.07$ & 0.13 & 0.14 & $0.07-0.21$ & $<0.001$ & $<0.001$ \\
\hline $\begin{array}{l}\text { BP and infarct } \\
\text { volume growth }^{\mathrm{b}}\end{array}$ & Coefficient & $95 \% \mathrm{CI}$ & $\mathrm{P}$ & Coefficient & $95 \% \mathrm{CI}$ & $\mathrm{P}$ & \\
\hline SBP per 10mmHg & 0.01 & $0.007-0.013$ & $<0.001$ & 0.003 & $-0.003-0.008$ & 0.85 & 0.001 \\
\hline DBP per $10 \mathrm{mmHg}$ & 0.01 & $0.006-0.016$ & $<0.001$ & -0.014 & $-0.025--0.004$ & 0.01 & $<0.001$ \\
\hline MAP per $10 \mathrm{mmHg}$ & 0.013 & $0.009-0.018$ & $<0.001$ & -0.005 & $-0.01--0.004$ & 0.26 & $<0.001$ \\
\hline BP and $m R S ~ 0-1^{c}$ & OR & $95 \% \mathrm{CI}$ & $\mathrm{P}$ & OR & $95 \% \mathrm{CI}$ & $\mathrm{P}$ & \\
\hline SBP per $10 \mathrm{mmHg}$ & 0.84 & $0.72-0.99$ & 0.03 & 0.95 & $0.71-1.29$ & 0.76 & 0.30 \\
\hline DBP per $10 \mathrm{mmHg}$ & 0.83 & $0.65-1.06$ & 0.14 & 1.78 & $1.004-3.15$ & 0.048 & 0.04 \\
\hline MAP per $10 \mathrm{mmHg}$ & 0.80 & $0.63-1.00$ & 0.05 & 1.38 & $0.82-2.36$ & 0.23 & 0.052 \\
\hline
\end{tabular}

For patients without reperfusion, higher BP was associated with better collateral flow, but increased infarct volume growth, and decreased odds of mRS 0-1.

For patients with reperfusion, higher BP was associated with better collateral flow, decreased infarct volume growth and increased odds of mRS 0-1.

Multivariate-adjusted for age, sex, baseline NIHSS, baseline glucose, history of hypertension, prior stroke, taking antiplatelet before admission, cause of stroke

Adjusted for age, sex, baseline NIHSS, baseline glucose, history of hypertension, endovascular treatment, occlusion/stenosis of ICA/M1, baseline ischemic core volume, baseline ischemic penumbra volume, MRI vs NCCT

${ }^{c}$ Adjusted for age, sex, baseline NIHSS, history of hypertension, prior stroke, taking antiplatelet before admission, reperfusion therapy, ischemic core volume, $\mathrm{PH} 2$

Abbreviations: BP blood pressure; SBP systolic blood pressure; DBP diastolic blood pressure; MAP mean arterial pressure; DT delay time; mRS modified Rankin Scale; NIHSS National Institutes of Health Stroke Scale; ICA internal carotid artery; M1 M1 segment of middle cerebral artery; PH2 parenchymal hematoma type 2, NCCT Non-Contrast Computed Tomography, MRI Magnetic Resonance Imaging 


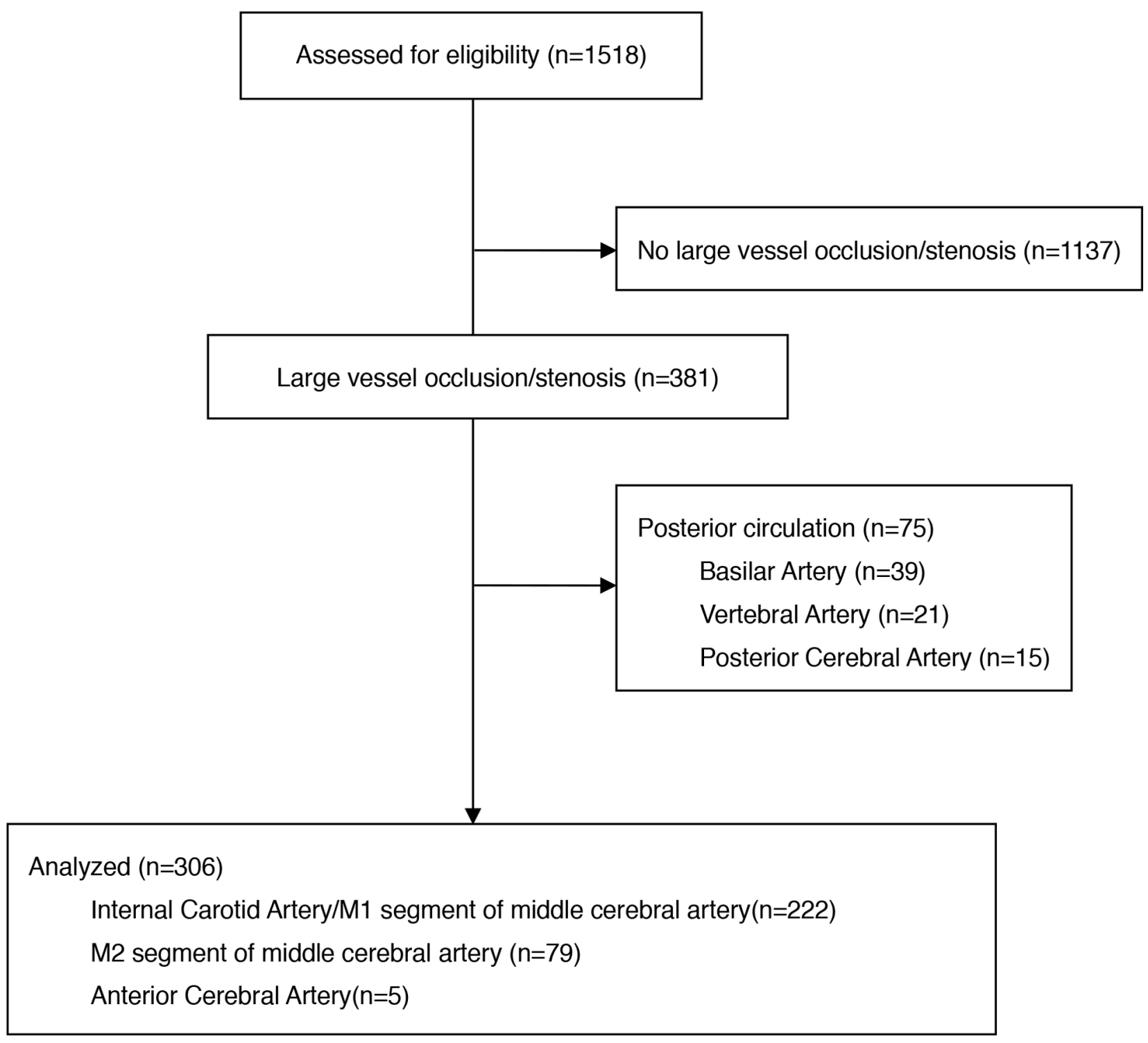

Figure 1 Flow diagram for patient selection

ANA_25428_Figure 1.tif

This article is protected by copyright. All rights reserved. 


\section{Please wait...}

If this message is not eventually replaced by the proper contents of the document, your PDF viewer may not be able to display this type of document.

You can upgrade to the latest version of Adobe Reader for Windows®, Mac, or Linux® by visiting http://www.adobe.com/go/reader_download.

For more assistance with Adobe Reader visit http://www.adobe.com/go/acrreader.

Windows is either a registered trademark or a trademark of Microsoft Corporation in the United States and/or other countries. Mac is a trademark
of Apple Inc., registered in the United States and other countries. Linux is the registered trademark of Linus Torvalds in the U.S. and other countries. 


\section{Please wait...}

If this message is not eventually replaced by the proper contents of the document, your PDF viewer may not be able to display this type of document.

You can upgrade to the latest version of Adobe Reader for Windows®, Mac, or Linux® by visiting http://www.adobe.com/go/reader_download.

For more assistance with Adobe Reader visit http://www.adobe.com/go/acrreader.

Windows is either a registered trademark or a trademark of Microsoft Corporation in the United States and/or other countries. Mac is a trademark
of Apple Inc., registered in the United States and other countries. Linux is the registered trademark of Linus Torvalds in the U.S. and other countries. 


\section{Please wait...}

If this message is not eventually replaced by the proper contents of the document, your PDF viewer may not be able to display this type of document.

You can upgrade to the latest version of Adobe Reader for Windows®, Mac, or Linux® by visiting http://www.adobe.com/go/reader_download.

For more assistance with Adobe Reader visit http://www.adobe.com/go/acrreader.

Windows is either a registered trademark or a trademark of Microsoft Corporation in the United States and/or other countries. Mac is a trademark
of Apple Inc., registered in the United States and other countries. Linux is the registered trademark of Linus Torvalds in the U.S. and other countries. 


\section{Please wait...}

If this message is not eventually replaced by the proper contents of the document, your PDF viewer may not be able to display this type of document.

You can upgrade to the latest version of Adobe Reader for Windows®, Mac, or Linux® by visiting http://www.adobe.com/go/reader_download.

For more assistance with Adobe Reader visit http://www.adobe.com/go/acrreader.

Windows is either a registered trademark or a trademark of Microsoft Corporation in the United States and/or other countries. Mac is a trademark
of Apple Inc., registered in the United States and other countries. Linux is the registered trademark of Linus Torvalds in the U.S. and other countries. 


\section{Please wait...}

If this message is not eventually replaced by the proper contents of the document, your PDF viewer may not be able to display this type of document.

You can upgrade to the latest version of Adobe Reader for Windows®, Mac, or Linux® by visiting http://www.adobe.com/go/reader_download.

For more assistance with Adobe Reader visit http://www.adobe.com/go/acrreader.

Windows is either a registered trademark or a trademark of Microsoft Corporation in the United States and/or other countries. Mac is a trademark
of Apple Inc., registered in the United States and other countries. Linux is the registered trademark of Linus Torvalds in the U.S. and other countries. 


\section{Please wait...}

If this message is not eventually replaced by the proper contents of the document, your PDF viewer may not be able to display this type of document.

You can upgrade to the latest version of Adobe Reader for Windows®, Mac, or Linux® by visiting http://www.adobe.com/go/reader_download.

For more assistance with Adobe Reader visit http://www.adobe.com/go/acrreader.

Windows is either a registered trademark or a trademark of Microsoft Corporation in the United States and/or other countries. Mac is a trademark
of Apple Inc., registered in the United States and other countries. Linux is the registered trademark of Linus Torvalds in the U.S. and other countries. 


\section{Please wait...}

If this message is not eventually replaced by the proper contents of the document, your PDF viewer may not be able to display this type of document.

You can upgrade to the latest version of Adobe Reader for Windows®, Mac, or Linux® by visiting http://www.adobe.com/go/reader_download.

For more assistance with Adobe Reader visit http://www.adobe.com/go/acrreader.

Windows is either a registered trademark or a trademark of Microsoft Corporation in the United States and/or other countries. Mac is a trademark
of Apple Inc., registered in the United States and other countries. Linux is the registered trademark of Linus Torvalds in the U.S. and other countries. 


\section{Please wait...}

If this message is not eventually replaced by the proper contents of the document, your PDF viewer may not be able to display this type of document.

You can upgrade to the latest version of Adobe Reader for Windows®, Mac, or Linux® by visiting http://www.adobe.com/go/reader_download.

For more assistance with Adobe Reader visit http://www.adobe.com/go/acrreader.

Windows is either a registered trademark or a trademark of Microsoft Corporation in the United States and/or other countries. Mac is a trademark
of Apple Inc., registered in the United States and other countries. Linux is the registered trademark of Linus Torvalds in the U.S. and other countries. 


\section{Please wait...}

If this message is not eventually replaced by the proper contents of the document, your PDF viewer may not be able to display this type of document.

You can upgrade to the latest version of Adobe Reader for Windows®, Mac, or Linux® by visiting http://www.adobe.com/go/reader_download.

For more assistance with Adobe Reader visit http://www.adobe.com/go/acrreader.

Windows is either a registered trademark or a trademark of Microsoft Corporation in the United States and/or other countries. Mac is a trademark
of Apple Inc., registered in the United States and other countries. Linux is the registered trademark of Linus Torvalds in the U.S. and other countries. 


\section{Please wait...}

If this message is not eventually replaced by the proper contents of the document, your PDF viewer may not be able to display this type of document.

You can upgrade to the latest version of Adobe Reader for Windows®, Mac, or Linux® by visiting http://www.adobe.com/go/reader_download.

For more assistance with Adobe Reader visit http://www.adobe.com/go/acrreader.

Windows is either a registered trademark or a trademark of Microsoft Corporation in the United States and/or other countries. Mac is a trademark
of Apple Inc., registered in the United States and other countries. Linux is the registered trademark of Linus Torvalds in the U.S. and other countries. 


\section{Please wait...}

If this message is not eventually replaced by the proper contents of the document, your PDF viewer may not be able to display this type of document.

You can upgrade to the latest version of Adobe Reader for Windows®, Mac, or Linux® by visiting http://www.adobe.com/go/reader_download.

For more assistance with Adobe Reader visit http://www.adobe.com/go/acrreader.

Windows is either a registered trademark or a trademark of Microsoft Corporation in the United States and/or other countries. Mac is a trademark
of Apple Inc., registered in the United States and other countries. Linux is the registered trademark of Linus Torvalds in the U.S. and other countries. 


\section{Please wait...}

If this message is not eventually replaced by the proper contents of the document, your PDF viewer may not be able to display this type of document.

You can upgrade to the latest version of Adobe Reader for Windows®, Mac, or Linux® by visiting http://www.adobe.com/go/reader_download.

For more assistance with Adobe Reader visit http://www.adobe.com/go/acrreader.

Windows is either a registered trademark or a trademark of Microsoft Corporation in the United States and/or other countries. Mac is a trademark
of Apple Inc., registered in the United States and other countries. Linux is the registered trademark of Linus Torvalds in the U.S. and other countries. 


\section{Please wait...}

If this message is not eventually replaced by the proper contents of the document, your PDF viewer may not be able to display this type of document.

You can upgrade to the latest version of Adobe Reader for Windows®, Mac, or Linux® by visiting http://www.adobe.com/go/reader_download.

For more assistance with Adobe Reader visit http://www.adobe.com/go/acrreader.

Windows is either a registered trademark or a trademark of Microsoft Corporation in the United States and/or other countries. Mac is a trademark
of Apple Inc., registered in the United States and other countries. Linux is the registered trademark of Linus Torvalds in the U.S. and other countries. 


\section{Please wait...}

If this message is not eventually replaced by the proper contents of the document, your PDF viewer may not be able to display this type of document.

You can upgrade to the latest version of Adobe Reader for Windows®, Mac, or Linux® by visiting http://www.adobe.com/go/reader_download.

For more assistance with Adobe Reader visit http://www.adobe.com/go/acrreader.

Windows is either a registered trademark or a trademark of Microsoft Corporation in the United States and/or other countries. Mac is a trademark
of Apple Inc., registered in the United States and other countries. Linux is the registered trademark of Linus Torvalds in the U.S. and other countries. 
Table 1. The association between BP and collateral flow parameters

\begin{tabular}{|c|c|c|c|c|c|c|}
\hline & \multicolumn{3}{|c|}{ Unadjusted } & \multicolumn{3}{|c|}{ Multivariate adjusted } \\
\hline & Coefficient & $95 \% \mathrm{CI}$ & $\mathrm{P}$ & Coefficient & $95 \% \mathrm{CI}$ & $\mathrm{P}$ \\
\hline \multicolumn{7}{|l|}{$\mathrm{DT}>3 \mathrm{~s} / \mathrm{DT}>6 \mathrm{~s}^{\mathrm{a}}$} \\
\hline SBP per 10mmHg & 0.04 & $0.02-0.06$ & $<0.001$ & 0.05 & $0.03-0.07$ & $<0.001$ \\
\hline DBP per 10mmHg & 0.07 & $0.04-0.11$ & $<0.001$ & 0.07 & $0.04-0.11$ & $<0.001$ \\
\hline MAP per $10 \mathrm{mmHg}$ & 0.07 & $0.04-0.10$ & $<0.001$ & 0.08 & $0.05-0.11$ & $<0.001$ \\
\hline \multicolumn{7}{|l|}{ Core volume $^{b}$} \\
\hline SBP per 10mmHg & -0.007 & $-0.01--0.0006$ & 0.03 & -0.02 & $-0.04--0.02$ & $<0.001$ \\
\hline DBP per 10mmHg & -0.02 & $-0.03--0.01$ & $<0.001$ & -0.06 & $-0.07--0.05$ & $<0.001$ \\
\hline MAP per $10 \mathrm{mmHg}$ & -0.07 & $-0.04--0.10$ & $<0.001$ & -0.06 & $-0.07--0.04$ & $<0.001$ \\
\hline \multicolumn{7}{|l|}{ DT $>3$ s lesion ${ }^{c}$} \\
\hline SBP per 10mmHg & -0.004 & $-0.002-0.01$ & 0.07 & -0.005 & $-0.01--0.0006$ & 0.03 \\
\hline DBP per 10mmHg & -0.01 & $-0.002--0.02$ & 0.01 & -0.02 & $-0.03--0.01$ & $<0.001$ \\
\hline MAP per $10 \mathrm{mmHg}$ & -0.01 & $-0.003--0.02$ & 0.005 & -0.02 & $-0.02--0.01$ & $<0.001$ \\
\hline
\end{tabular}

Increasing BP was significantly associated with increase in $\mathrm{DT}>3 \mathrm{~s} / \mathrm{DT}>6 \mathrm{~s}$ ratio, and decrease in core, DT $>3 \mathrm{~s}$, DT $>6 \mathrm{~s}$ volume.

a Multivariate-adjusted for age, sex, baseline NIHSS, baseline glucose, history of hypertension, prior stroke, taking antiplatelet before admission, cause of stroke

b Multivariate-adjusted for age, sex, baseline NIHSS, baseline glucose, history of hypertension, prior stroke, taking antiplatelet before admission, occlusion/stenosis of ICA/M1

c Multivariate-adjusted for age, sex, baseline NIHSS, baseline glucose, history of hypertension, prior stroke, taking antiplatelet before admission, history of dyslipidemia, occlusion/stenosis of ICA/M1

Abbreviations: BP blood pressure; SBP systolic blood pressure; DBP diastolic blood pressure; MAP mean arterial pressure; DT delay time; NIHSS National Institutes of Health Stroke Scale; ICA internal carotid artery; M1 M1 segment of middle cerebral artery 
Table 2. The association between BP and outcomes

\begin{tabular}{|c|c|c|c|c|c|c|c|c|c|}
\hline \multirow[b]{2}{*}{ BP and mRS 0-1 } & \multicolumn{3}{|c|}{ Unadjusted } & \multicolumn{3}{|c|}{ Sex- and age- adjusted } & \multicolumn{3}{|c|}{ Multivariate adjusted } \\
\hline & OR & $95 \% \mathrm{CI}$ & $P$ & OR & $95 \% \mathrm{CI}$ & $\mathrm{P}$ & $\mathrm{OR}^{\mathrm{a}}$ & $95 \% \mathrm{CI}^{\mathrm{a}}$ & $\mathrm{P}^{\mathrm{a}}$ \\
\hline SBP per 10mmHg & 0.84 & $0.76-0.93$ & 0.001 & 0.85 & $0.76-0.95$ & 0.003 & 0.88 & $\begin{array}{c}0.78- \\
0.995\end{array}$ & 0.048 \\
\hline DBP per 10mmHg & 0.96 & $0.82-1.13$ & 0.64 & 0.89 & $0.75-1.05$ & 0.17 & 0.93 & $\begin{array}{c}0.77- \\
1.14\end{array}$ & 0.49 \\
\hline MAP per $10 \mathrm{mmHg}$ & 0.86 & $0.74-1.00$ & 0.050 & 0.82 & $0.71-0.97$ & 0.02 & 0.88 & $\begin{array}{c}0.74- \\
1.05\end{array}$ & 0.16 \\
\hline $\begin{array}{l}\text { BP and infarct } \\
\text { volume growth }\end{array}$ & Coefficient & $95 \% \mathrm{CI}$ & $\mathrm{P}$ & Coefficient & $95 \% \mathrm{CI}$ & $\mathrm{P}$ & Coefficient $^{\mathrm{b}}$ & $95 \% \mathrm{CI}^{\mathrm{b}}$ & $\mathrm{P}^{\mathrm{b}}$ \\
\hline SBP per $10 \mathrm{mmHg}$ & 0.010 & $\begin{array}{c}0.007- \\
0.013\end{array}$ & $<0.001$ & 0.010 & $\begin{array}{ll}0.008 & - \\
0.012 & \end{array}$ & $<0.001$ & 0.009 & $\begin{array}{c}0.006- \\
0.012\end{array}$ & $<0.001$ \\
\hline DBP per $10 \mathrm{mmHg}$ & 0.012 & $\begin{array}{c}0.008- \\
0.015\end{array}$ & $<0.001$ & 0.014 & $\begin{array}{c}0.010- \\
0.018\end{array}$ & $<0.001$ & 0.008 & $\begin{array}{c}0.004- \\
0.011\end{array}$ & $<0.001$ \\
\hline MAP per $10 \mathrm{mmHg}$ & 0.015 & $\begin{array}{c}0.011 \text { - } \\
0.018\end{array}$ & $<0.001$ & 0.015 & $\begin{array}{c}0.012- \\
0.019\end{array}$ & $<0.001$ & 0.011 & $\begin{array}{c}0.008- \\
0.015\end{array}$ & $<0.001$ \\
\hline $\mathrm{BP}$ and PH2 & OR & $95 \% \mathrm{CI}$ & $\mathrm{P}$ & OR & $95 \% \mathrm{CI}$ & $\mathrm{P}$ & $\mathrm{OR}^{\mathrm{c}}$ & $95 \% \mathrm{CI}^{\mathrm{c}}$ & $\mathrm{P}^{\mathrm{c}}$ \\
\hline SBP per $10 \mathrm{mmHg}$ & 1.00 & $0.82-1.23$ & 0.94 & 1.00 & $0.82-1.23$ & 0.97 & 0.94 & $\begin{array}{c}0.76- \\
1.17\end{array}$ & 0.59 \\
\hline DBP per 10mmHg & 1.02 & $0.73-1.41$ & 0.92 & 1.06 & $0.77-1.47$ & 0.72 & 0.97 & $\begin{array}{c}0.68- \\
1.39\end{array}$ & 0.89 \\
\hline MAP per $10 \mathrm{mmHg}$ & 1.02 & $0.75-1.38$ & 0.91 & 1.04 & $0.77-1.41$ & 0.80 & 0.94 & $\begin{array}{c}0.68- \\
1.31\end{array}$ & 0.73 \\
\hline
\end{tabular}

Higher BP was associated with decreased odds of an excellent functional outcome (mRS 0-1) and increased infarct volume growth.

a Adjusted for age, sex, baseline NIHSS, history of hypertension, prior stroke, taking antiplatelet before admission, reperfusion therapy, ischemic core volume, PH2

${ }^{\mathrm{b}}$ Adjusted for age, sex, baseline NIHSS, baseline glucose, endovascular treatment, occlusion/stenosis of ICA/M1, baseline ischemic core volume, baseline ischemic penumbra volume, MRI vs NCCT

${ }^{\text {c }}$ Adjusted for age, sex, baseline NIHSS, taking antiplatelet before admission, reperfusion therapy

Abbreviations: BP blood pressure; mRS modified Rankin Scale; SBP systolic blood pressure; DBP diastolic blood pressure; MAP mean arterial pressure; OR odds ratio; NIHSS National Institutes of Health Stroke Scale; ICA internal carotid artery; M1 M1 segment of middle cerebral artery; PH2 parenchymal hematoma type 2, NCCT Non-Contrast Computed Tomography, MRI Magnetic Resonance Imaging 
Table 3. The association between BP, mRS 0-1, infarct volume growth and DT $>3 \mathrm{~s} / \mathrm{DT}>6$ s ratio in subgroup analysis (multivariate-adjusted)

\begin{tabular}{|l|c|c|c|c|c|c|c|}
\hline & \multicolumn{3}{|c|}{ Patients without reperfusion (n=204) } & \multicolumn{3}{|c|}{ Patients with reperfusion(n=68) } & P for interaction \\
\hline BP and & Coefficient & $95 \%$ CI & P & Coefficient & $95 \%$ CI & P & \\
\hline DT $>$ 3s/DT $>\mathbf{6} \mathbf{s}^{\mathrm{a}}$ & & & & & & & \\
\hline SBP per 10mmHg & 0.03 & $0.004-0.057$ & 0.02 & 0.08 & $0.04-0.12$ & $<0.001$ & 0.02 \\
\hline
\end{tabular}




\begin{tabular}{|c|c|c|c|c|c|c|c|}
\hline DBP per 10mmHg & 0.03 & $-0.03-0.05$ & 0.63 & 0.13 & $0.05-0.20$ & 0.001 & $<0.001$ \\
\hline MAP per 10mmHg & 0.03 & $-0.01-0.07$ & 0.13 & 0.14 & $0.07-0.21$ & $<0.001$ & $<0.001$ \\
\hline $\begin{array}{l}\text { BP and infarct } \\
\text { volume growth }\end{array}$ & Coefficient & $95 \%$ CI & P & Coefficient & $95 \%$ CI & P & \\
\hline SBP per 10mmHg & 0.01 & $0.007-0.013$ & $<0.001$ & 0.003 & $-0.003-0.008$ & 0.85 & 0.001 \\
\hline DBP per 10mmHg & 0.01 & $0.006-0.016$ & $<0.001$ & -0.014 & $-0.025--0.004$ & 0.01 & $<0.001$ \\
\hline MAP per 10mmHg & 0.013 & $0.009-0.018$ & $<0.001$ & -0.005 & $-0.01--0.004$ & 0.26 & $<0.001$ \\
\hline BP and mRS 0-1 & OR & $95 \% C I$ & P & OR & $95 \%$ CI & P & \\
\hline SBP per 10mmHg & 0.84 & $0.72-0.99$ & 0.03 & 0.95 & $0.71-1.29$ & 0.76 & 0.30 \\
\hline DBP per 10mmHg & 0.83 & $0.65-1.06$ & 0.14 & 1.78 & $1.004-3.15$ & 0.048 & 0.04 \\
\hline MAP per 10mmHg & 0.80 & $0.63-1.00$ & 0.05 & 1.38 & $0.82-2.36$ & 0.23 & 0.052 \\
\hline
\end{tabular}

For patients without reperfusion, higher BP was associated with better collateral flow, but increased infarct volume growth, and decreased odds of mRS 0-1.

For patients with reperfusion, higher BP was associated with better collateral flow, decreased infarct volume growth and increased odds of mRS 0-1.

Multivariate-adjusted for age, sex, baseline NIHSS, baseline glucose, history of hypertension, prior stroke, taking antiplatelet before admission, cause of stroke

Adjusted for age, sex, baseline NIHSS, baseline glucose, history of hypertension, endovascular treatment, occlusion/stenosis of ICA/M1, baseline ischemic core volume, baseline ischemic penumbra volume, MRI vs NCCT

Adjusted for age, sex, baseline NIHSS, history of hypertension, prior stroke, taking antiplatelet before admission, reperfusion therapy, ischemic core volume, $\mathrm{PH} 2$

Abbreviations: BP blood pressure; SBP systolic blood pressure; DBP diastolic blood pressure; MAP mean arterial pressure; DT delay time; mRS modified Rankin Scale; NIHSS National Institutes of Health Stroke Scale; ICA internal carotid artery; M1 M1 segment of middle cerebral artery; PH2 parenchymal hematoma type 2, NCCT Non-Contrast Computed Tomography, MRI Magnetic Resonance Imaging 


\section{University Library}

\section{- M M N E R VA A gateway to Melbourne's research publications}

Minerva Access is the Institutional Repository of The University of Melbourne

Author/s:

Hong, L;Cheng, X;Lin, L;Bivard, A;Ling, Y;Butcher, K;Dong, Q;Parsons, M;Spratt, N;Levi, C;Choi, PMC;Kleining, T;O'Brien, B;Lou, M

Title:

The blood pressure paradox in acute ischemic stroke

Date:

2019-03-01

Citation:

Hong, L., Cheng, X., Lin, L., Bivard, A., Ling, Y., Butcher, K., Dong, Q., Parsons, M., Spratt, N., Levi, C., Choi, P. M. C., Kleining, T., O'Brien, B. \& Lou, M. (2019). The blood pressure paradox in acute ischemic stroke. ANNALS OF NEUROLOGY, 85 (3), pp.331-339. https:// doi.org/10.1002/ana.25428.

Persistent Link:

http://hdl.handle.net/11343/285416 